\title{
The wireless energy transfer (WET) using ultra high frequency (UHF) for human body implant recharging
}

\author{
Fahmy Rinanda Saputri ${ }^{1}$, Sunarno $^{2^{*}}$, Memory Motivanisman Waruwu ${ }^{3}$, Rony Wijaya ${ }^{4}$ \\ ${ }^{1}$ Master Student in Department of Nuclear Engineering and Engineering Physics, Faculty of Engineering, Universitas Gadjah Mada \\ ${ }^{2,3}$ Department of Nuclear Engineering and Engineering Physics, Faculty of Engineering, Universitas Gadjah Mada \\ ${ }^{4}$ Production House Unit, Faculty of Medicine, Universitas Gadjah Mada
}

\begin{abstract}
The wireless energy transfer (wet) is widely used in many fields. In particularly for medical, this technique can be implemented to an electronic human body implant recharging. The possible size and shape of the receiver antenna in the experiment before still become a problem. Using Yagi-Uda model antenna for the transmitter unit in ultra-high frequency (UHF) band, this paper refers to design a receiver antenna for implemented as a human implant recharging. The receiver antenna has successfully designed in minimal with its size and shape of the designed antenna is about $1.44 \mathrm{~cm}^{3}$.
\end{abstract}

\section{Introduction}

The wireless energy transfer (wet) has been carried out in various field. Sunarno has designed the basic system for the wireless energy transfer based on ultra high frequency (UHF) band [1]. The transmitter and receiver system was using parabolic metal reflector system. This system able to transfer energy and fill the rechargeable battery with the efficiency was about $1 \%$.

Sunarno and the team have also succeeded in their experiment about transferring energy using wireless technology based on UHF band with Yagi-Uda Directional antenna as a transmitter antenna. From the experiment can be concluded that distance and angle of transmitter antenna influenced for the amount of energy received. In this case, the amount of energy received is proportional with the current and voltage measured [2].

Reference [3] gave an experiment about designing ultra wideband antenna. It's application for a short distance communication system. The performance of the antenna is good with its fractional bandwidth of more than $108.2 \%$ and the range antenna resonance frequency from 3.1 to $10.5 \mathrm{GHz}$. In [4], the author presented elaborated prototype of energy harvesting in the ultrahigh frequency band. It is used for excess energy and conducting radio communication process.

For implantable Bioelectronics, wireless energy transfer is possible to be applied. Reference [5] reviews the theory, link designed, and different wireless strategy ranging from the near-field inductive and capacitive coupling to the ultrasonics, mid-field and far field for the neural implants. The author considered that wirelessly powered implants are efficient and integrable if it is implemented as implantation device.

Recent various contributions in the area of energy harvesting are summarized in [6]. Based on the author's reviews, there is interdisciplinary nature from the devices that wireless transfer and harvest energy. Additionally, in reference [7], the authors discuss issue and challenge in future implementing the integration of wireless charging technologies.
Exiting the various applications of the wireless energy transfer, this paper that continuing the research before [2] was conducting research specifically for the medical area. In the medical, the wet can be utilized as an electronic human body implant recharging. The methods have already done in the experiments before, but there are problems, such as the size of the receiver unit doesn't match if it is implemented as an implant and can't be applied as an energy harvesting yet. An energy harvesting technique is very advantageous because recharging energy can occur automatically if the people who use implant are in the radiation electromagnetic field and should not be done intensively in the hospital.

The paper is organized into five sections. Section II discusses various terms explanation for understanding this research topic. The setup and methodological of the system concerns specifically in Section III. Furthermore, in Section IV, we discuss with a brief result clarifications based on the final graphs and data. We end with a conclusion and future research in the real receiver application for human body implant in Section V.

\section{Theoretical Background}

\subsection{Ultra high-frequency antenna}

Ultra high frequency is electromagnetic waves in the frequency of $300 \mathrm{MHz}$ to $3.000 \mathrm{MHz}$. The range of wave length is $10 \mathrm{~cm}$ to $100 \mathrm{~cm} \mathrm{[10].} \mathrm{The} \mathrm{popular} \mathrm{UHF}$ antenna is Yagi-Uda. Yagi-Uda antenna is used to transfers or receives the radio waves and has a directional function, meaning it only can take or receives the signal in single direction i.e. front. Yagi-Uda antenna has range of gain is 3 to $20 \mathrm{~dB}$. The elements of this antenna are driven, reflector, director, and boom [10]. Driven is the feed point to perform the transfer of power of the antenna [2]. The length of the driven antenna is $0.5 \lambda$. A reflector is utilized for reflecting the signal. The position is in the behind. The length of a reflector is about $0.55 \lambda$. Director as a referrer has the shorter length than driven. Adding director will increase the gain,

\footnotetext{
Corresponding author: sunarno@ugm.ac.id
} 
however, the beam pattern will more narrow. The boom is part of the antenna made of the wood and metal stem for placing driven, reflector, and director. The antenna has a space between elements is $0.1 \lambda$ of the frequency.

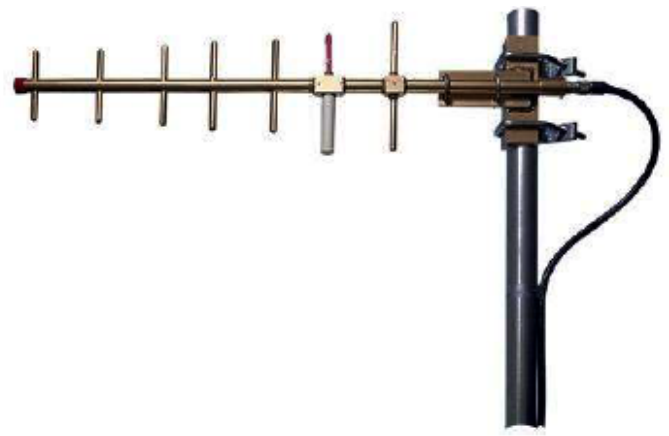

Fig. 1. The Yagi-Uda antenna [10]

\subsection{Wireless charging}

Wireless charging is the technology that enables a power to transmit electromagnetic energy to an electrical load without cable. Wireless charging has many advantages [7]: wireless charging improves user friendliness as without cable for implementation. better product durability renders the smaller devices design and fabrication and is more flexible and energy-efficient in an on-demand fashion. There are two major wireless charging technologies, i.e. radio frequency based wireless charging and coupling-based wireless charging. Wireless charging is attracting a many application, such as smartphone battery charging, vehicle charging, wireless communication, and biomedical devices [2]. For biomedical devices as an implantation, the couplingbased wireless charging has been conducted [11].

white, and this should be taken into account when preparing them.

\section{Experimental setup}

This study proposed that the use of the Yagi-Uda model antenna using ultra high frequency (UHF) is able to transfer energy with the wireless energy transfer technique. The wireless technique can be applied in many fields, especially in the medical field, that is to recharging electronic implant.

The methodology and technique of research have been conducted in previous research that was held by Sunarno et.al. For the transmitter unit, we also used Yagi-Uda model antenna. The beaming pattern of YagiUda antenna is the reason. It is very focused, moreover in the narrow spot area. The transmitter antenna is able to changes electron wave to electromagnetic wave to the receiver antenna.

The wet system has designed by using ultra-high frequency band, that is $430 \mathrm{MHz}$ as an energy carrier. Firstcom FR-488 instrument is used for measuring measured energy [2]. The power transmitter unit is 55 watt and 12 volts. The value of standing wave ratio in this research is 1.12 .
In this system, the same electronic scheme, the YagiUda Antenna, and the beam antenna were used. In previous research, that components were conducted by Sunarno et.al, are shown in Fig. 2, 3, 4, and 5.

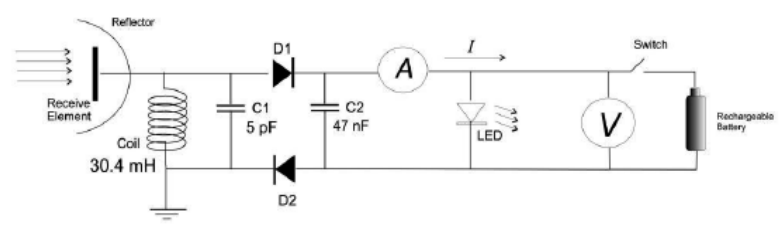

Fig. 2. The scheme of receiver circuit unit [1]

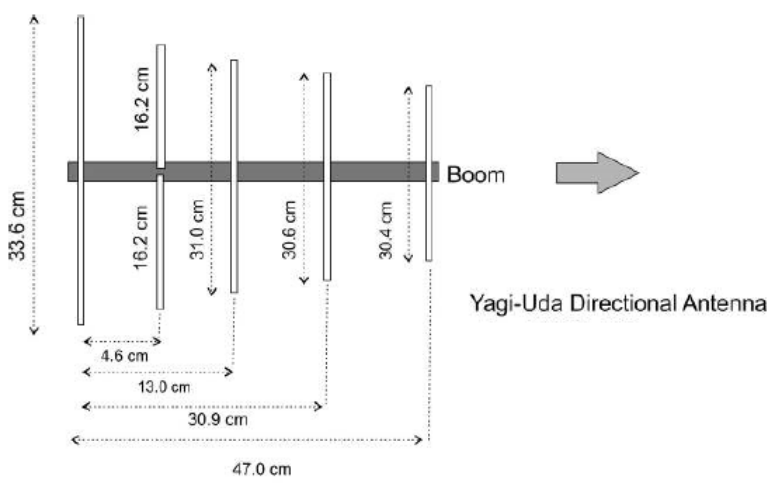

Fig. 3. The Yagi-Uda model antenna [2]

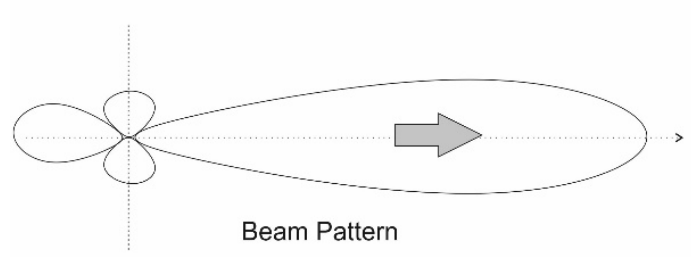

Fig. 4. The beam pattern of Yagi-Uda model antenna [2]

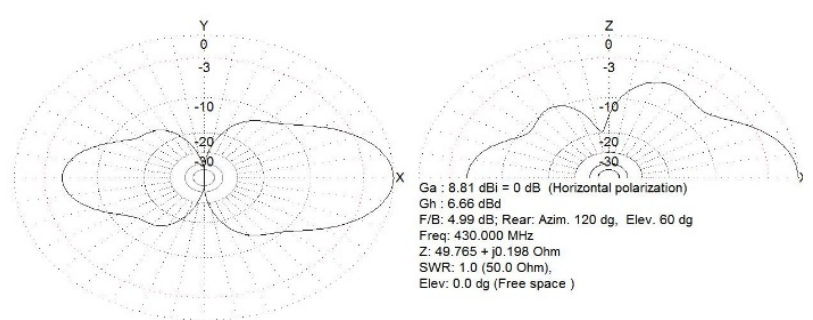

Fig. 5. The 2D prediction of beam pattern [2] 


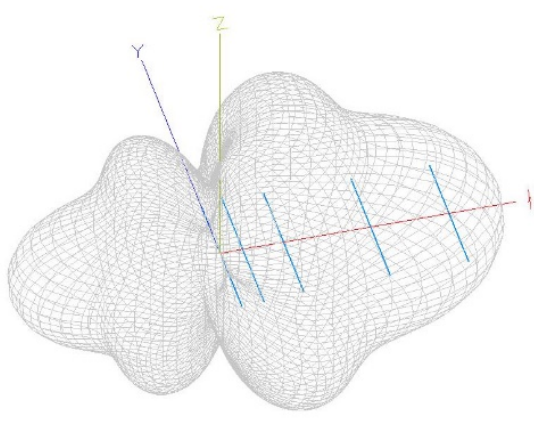

Fig. 6. The 3D prediction of beam pattern [2]
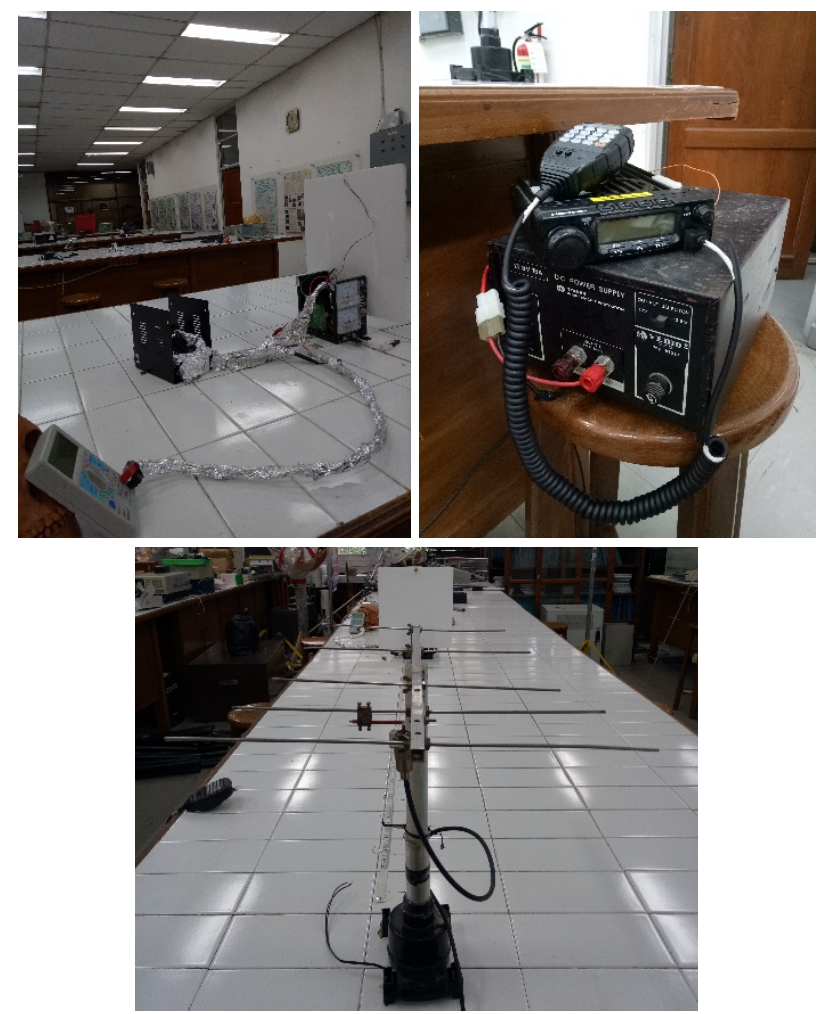

Fig. 7. The research devices

Fig. 6 shown the devices of wireless energy transfer system for this research. We used Yagi-Uda antenna of the transmitter unit, Firstcom FR-488 for measuring transmitter unit, voltmeter and ampere meter for measuring received energy and use the modified antenna as a receiver antenna. Because the implementation of the receiver antenna is for human implant electronic for recharging system, we design the minimal antenna. The size of the antenna is about $1.44 \mathrm{~cm}^{3}$. The size is able enough to insert in the body as human impant electronic. The receiver antenna is shown in Fig. 7.

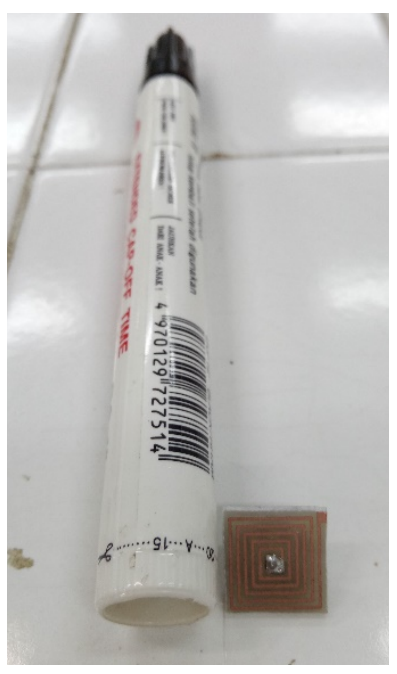

Fig. 8. The receiver antenna

\section{Result and discussion}

The study focused on the analysis of the unit receiver antenna design and doing experiment with varying the distance between transmitter and receiver antenna. The energy received based on varying distance is also analyzed. The measured result of the system based on varying distance is shown in Fig. 8.

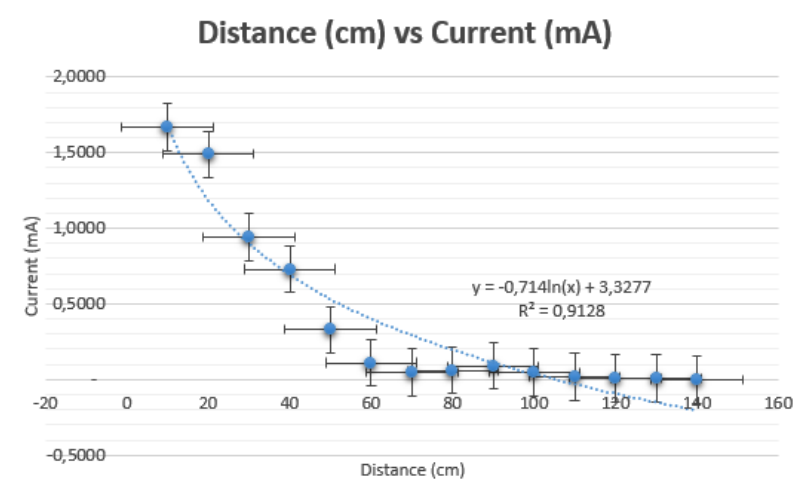

Fig. 9. Capture current versus distance with 55-watt energy source

The results show that the most of the current decreases when the distance enlarged between transmitter and receiver unit. The received power can be calculated by a formula,

$$
P=V \times I
$$




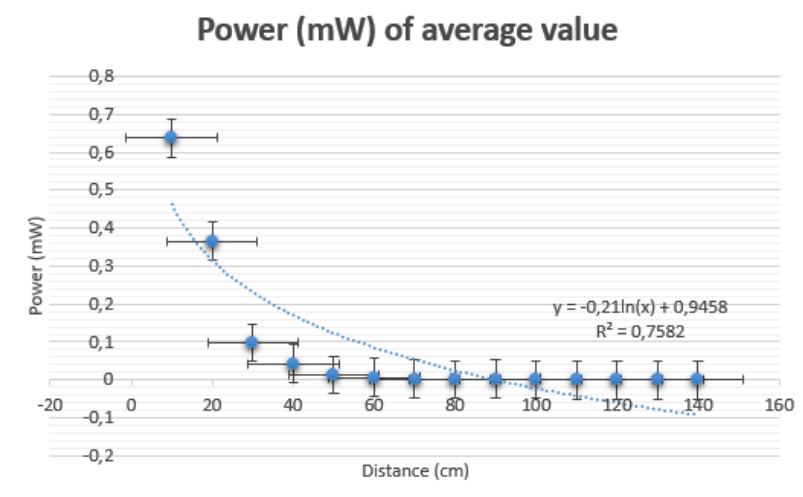

Fig. 10. Capture power versus distance with 55-watt energy source

The result of power in $\mathrm{mW}$ can be calculated by the measured voltage times the measured current in the receiver antenna. Fig. 9 is the graph capture of the received power. The technique was applied to transferring energy. The size of the receiver antenna that has been designed is $1.44 \mathrm{~cm}^{3}$. The technique can recharge implant device without doing surgery. The antenna receiver design is successful to receiving energy in the ultra high-frequency band with $0.00116 \%$ of the efficiency, $10 \mathrm{~cm}$ of the distance, using Yagi-Uda model antenna as a transmitter. The energy received by the constructed system was also higher with the short distance between transmitter and receiver antenna compared to the far distance.

In the next research, received antenna will be implemented to the deaf. Fig. 10 shows wireless energy transfer for human implantation system diagram that will be used in the next research, specifically for bone vibrator implantation system as in Fig. 11.

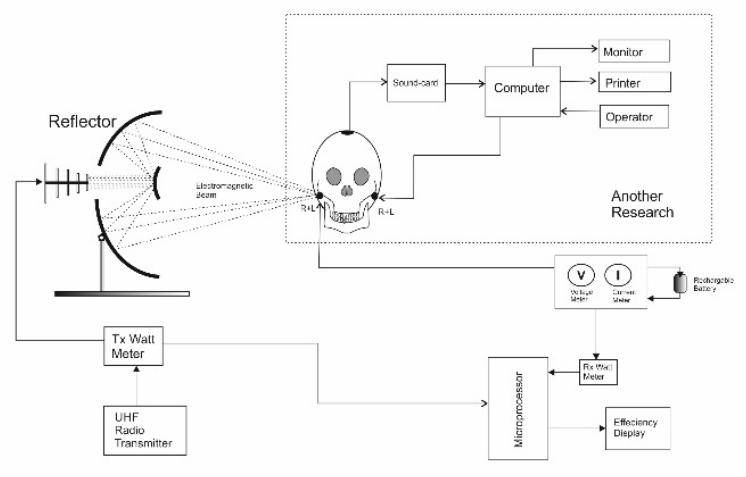

Fig. 11. The wireless energy transfer for human implantation research system diagram experiment system diagram

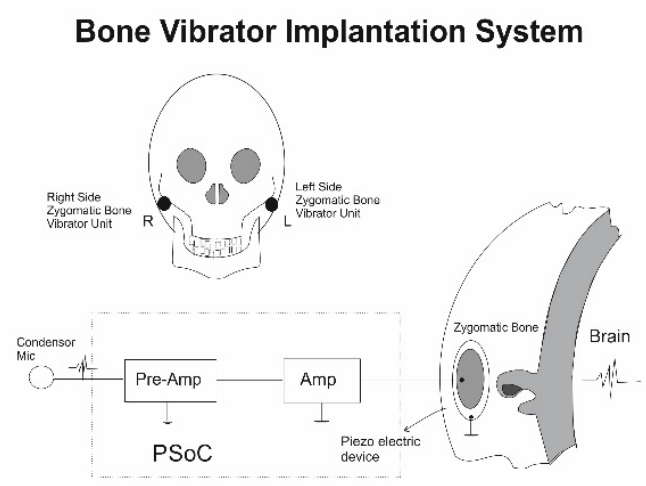

Fig. 12. Bone vibrator implantation system

\section{Bone Vibrator Monitoring System}

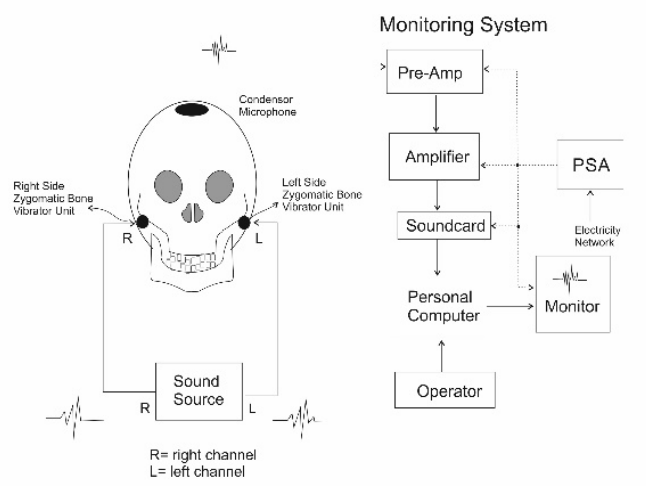

Fig. 13. Capture power versus distance with 55-watt energy source

Fig. 12 is the monitoring system of the final research. The final of the future research is implementation the wireless energy transfer using ultra-high frequency for the implantation electronic to the deaf. This electronic is able to help the deaf to feel the vibration of the sound and can monitor the vibrator.

\section{Conclusion}

The energy transfer was successfully performed using a wireless energy transfer technique using the ultra-high frequency (UHF) band with the Yagi-Uda model antenna as a transmitter and a small receiver antenna design, which is about $1.44 \mathrm{~cm}^{3}$. The research has succeeded transfer energy with the minimal design od the receiver antenna. This minimal antenna received size allows it to be implanted into the human body as an electronic implant recharging.

The authors would like to thank Sensor and Telecontrol System Laboratory, Department of Nuclear Engineering and Engineering Physics, Faculty of Engineering, Universitas Gadjah Mada for providing the lab facilities to complete this research. 


\section{References}

1. Sunarno, Astechnova, 62-70 (2014)

2. Sunarno, F.R. Saputri, M.M. Waruwu, R. Wijaya, International Conference on Science and Technology (2017)

3. V.K. Rai, M. Kumar, M.D. Upadhayay, International Conference on Computational Intelligence and Communication Networks, 16-19 (2015)

4. L. Bernacki, R. Gozdur, N. Salamon, Conference Series, 709 (2016)

5. K. Agarwal, R. Jegadeesan, Y-X. Guo, N. V. Thakor, IEEE Reviews in Biomedical Engineering, 10 (2017)

6. S. Ulukus, A. Yener, E. Erkip, O. Simeone, M. Zorzi, P. Grover, K. Huang, IEEE J. Selected Areas in Communications, 33 (2015)

7. X. Lu, P. Wang, D. Niyato, D. I. Kim, Z. Han, IEEE Comm. Surveys \& Tutorials, 18, 1413-1452 (2016)

8. L. Xie, Y. Shi, Y. T. Hou, W. Lou, IEEE Wireless Communications, 140-145 (2013)

9. P. Nepa, H. Rogier, IEEE Antennas \& Propagation Magazine, 30-52 (2015)

10. Sunarno, Diktat Mata Kuliah TKF 3515Komunikasi Data, Jurusan Teknik Fisika Fakultas Teknik Universitas Gadjah Mada (2013)

11. S. C. Tang, T. L. T. Lun, Z. Guo, K. W. Kwok, N. J. McDannold, IEEE Trans. Power Electronics 32, 3844-3857 (2017) 